\title{
Adaptive Control System for Autonomous Vehicle Path Following
}

\author{
Milan Stork \\ Department of Applied Electronics \\ and Telecommunications/RICE \\ University of West Bohemia \\ Plzen, Czech Republic \\ stork@kae.zcu.cz
}

\author{
Jiri Pinker \\ Department of Applied Electronics \\ and Telecommunications/RICE \\ University of West Bohemia \\ Plzen, Czech Republic \\ pinker@kae.zcu.cz
}

\author{
Petr Weissar \\ Department of Applied Electronics \\ and Telecommunications/RICE \\ University of West Bohemia \\ Plzen, Czech Republic \\ weissar@kae.zcu.cz
}

\begin{abstract}
Autonomy in vehicles is a rapidly expanding technology that is of interest in many major car companies. Autonomous driving enables safer journeys by removing human error in driving, as well as reducing driving time and fuel consumption by optimizing the engine and brake actuation. While certain autonomous functions in cars have been in use for over a decade, such as lane keeping and automatic parking these features are subject to specific scenarios. A fully automated vehicle needs to take into account the unpredictable and complex environments that cars drive in. The idea of a self-driving car in a city environment needs specific requirements such as reliable path following, accurate sensor data and safe drive. The scope of this paper is to create a simple path following solution which enables an autonomous car to accurately traverse along any given path.
\end{abstract}

Keywords - adaptive control; adaptive controller; continuous; digital; model reference; adaptive control

\section{INTRODUCTION}

A control system is a device that regulates or controls the dynamics systems or process. Adaptive control is one of the widely used control strategies to design advanced control systems which can change properties according to changed conditions. Model Reference Adaptive Control (MRAC) is a direct adaptive strategy with some adjustable controller parameters and an adjusting mechanism to adjust them. In this work the adaptive control is used for autonomous vehicle trajectory following with minimal error (see Fig.1).

\section{ADAPTIVE CONTROL}

On contrary to the well known and simple structured fixed gain, e.g. PID controllers, adaptive controllers are very effective to handle the unknown parameter variations and environmental changes. An adaptive controller usually consists of two loops, an outer loop or normal feedback loop and an inner loop

Milan Stork's participation was supported by Department of Applied Electronics and Telecommunications, University of West Bohemia, Plzen, Czech Republic and by the European Regional Development Fund and the Ministry of Education, Youth and Sports of the Czech Republic under the Regional Innovation Centre for Electrical Engineering (RICE), project No. LO1607 and by the Internal Grant Agency of University of West Bohemia in Plzen, the proiect SGS-2018-001 or parameter adjustment loop.

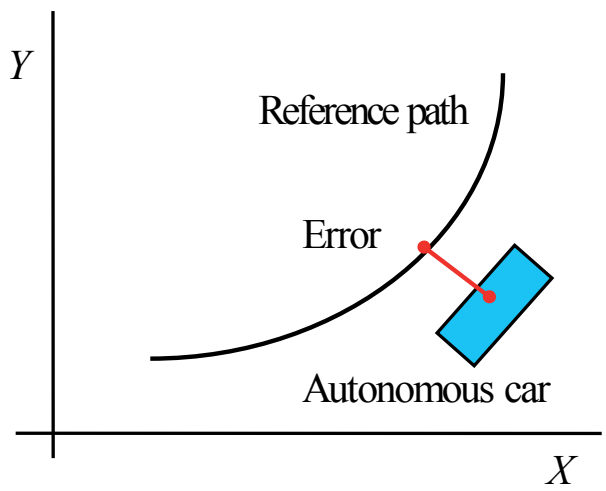

Figure 1. The principle of autonomous car trajectory folow

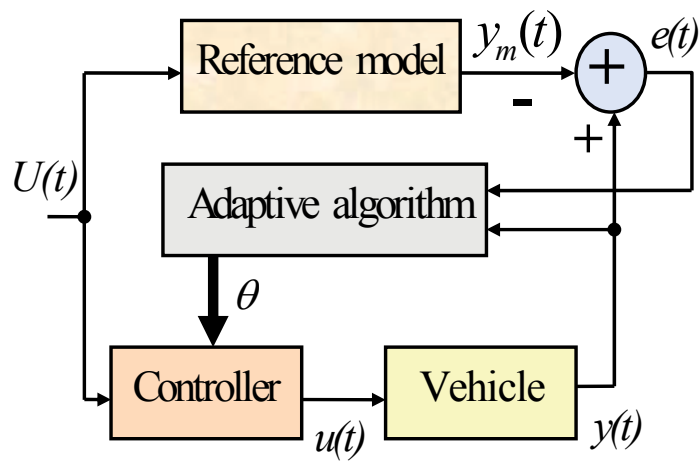

Figure 2. The block diagram of continuous adaptive system

The principle of MRAC is shown on Fig. 2. The output (position) of reference model (RM) is $y_{m}(t)$ and $y(t)$ is the output (position) of a vehicle or an autonomous car (ACA). The error between the output of RM and ACA is $e(t)$. The adaptive algorithm evaluates $y(t)$ and $e(t)$ and generates a vector of parameters $\theta$ for the controller. Adaptive control is based on MIT rules developed in 1960 by the researchers of Massachusetts Institute of Technology (MIT) and used to design the autopilot system for aircrafts [1 - 5].

The cost function (based on MIT rules [6-8]) is defined as 


$$
J(\theta)=e^{2} / 2=\left(y(t)-y_{m}(t)\right)^{2} / 2
$$

where $e$ is the output error, while $\theta$ is an adjustable vector which must be adjusted to minimize $J$. Let's assume $2^{\text {nd }}$ order linear MODEL $F_{m}(s)$ and VEHICLE $F(s)$ dynamics

$$
\begin{gathered}
F_{m}(s)=\frac{1}{s^{2}+1.41 s+1} \\
F(s)=\frac{0.5}{s^{2}+2 s+1}
\end{gathered}
$$

Step responses of the MODEL and VEHICLE are shown in Fig. 3.

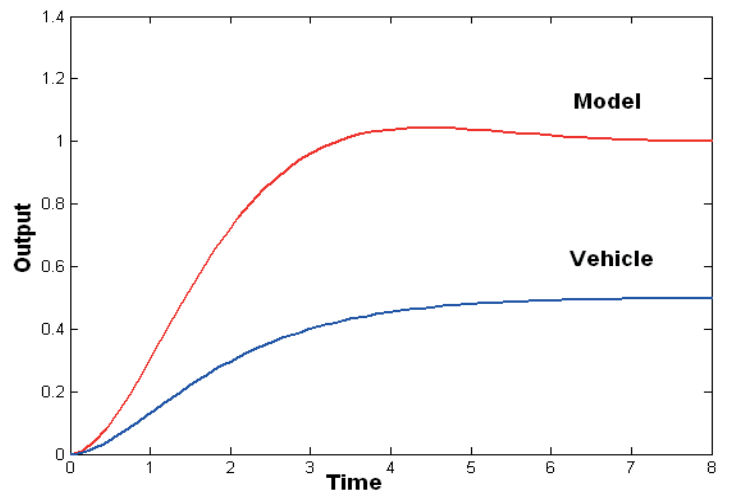

Figure 3. Step responses of MODEL and VEHICLE

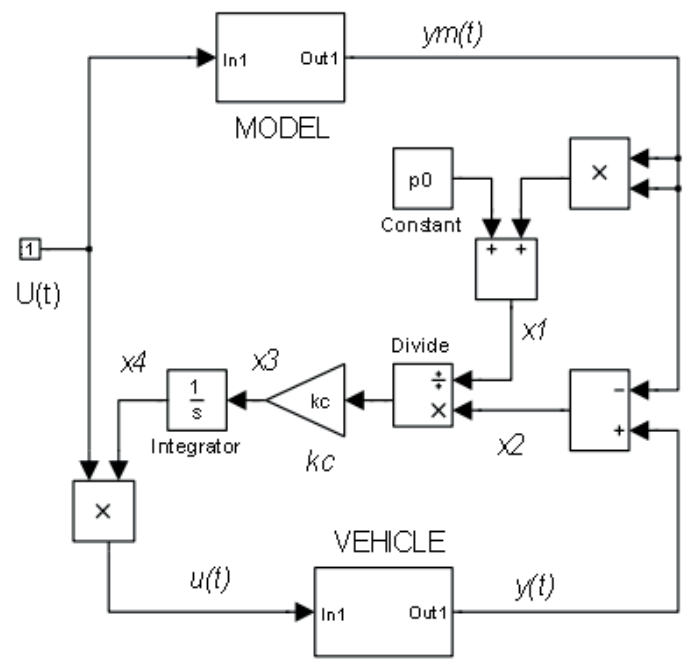

Figure 4. The block diagram of continuous adaptive system

The block diagram of continuous adaptive system is displayed in Fig. 4. The adaptive algorithm is designed according to following equations (4) - (8)

$$
x_{1}(t)=p_{0}+y_{m}^{2}
$$

where $p_{0}$ is small positive number $\left(p_{0}=0.01\right)$.

$$
x_{2}(t)=y_{m}\left(y-y_{m}\right)
$$

$$
x_{3}(t)=k_{C} \frac{x_{2}(t)}{x_{1}(t)}=k_{C} \frac{y_{m}\left(y-y_{m}\right)}{p_{0}+y_{m}^{2}}
$$

where $k_{C}$ is a gain of the controller (must be negative). The output of the adaptive algorithm is $x_{4}$, see eq. (7).

$$
x_{4}(t)=\int x_{3}(t) d t=k_{C} \int \frac{y_{m}\left(y-y_{m}\right)}{p_{0}+y_{m}^{2}} d t
$$

The control signal $U(t)$ (trajectory) modified by $x_{4}$ is given in eq. (8)

$$
\begin{aligned}
u(t) & =U(t) x_{4}(t) \\
& =U(t) \cdot k_{C} \int \frac{y_{m}\left(y-y_{m}\right)}{p_{0}+y_{m}^{2}} d t
\end{aligned}
$$

In Fig. 5 is presented the prescribed trajectory and MODEL trajectory

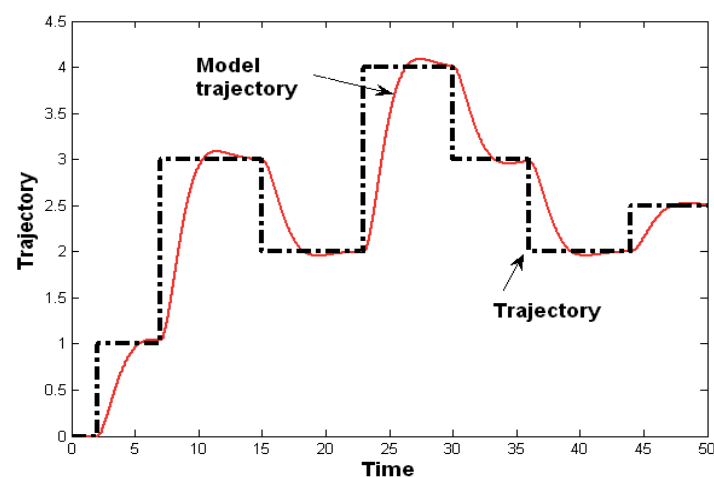

Figure 5. The prescribed trajectory (dash-dot, black) and MODEL trajectory (solid, red)

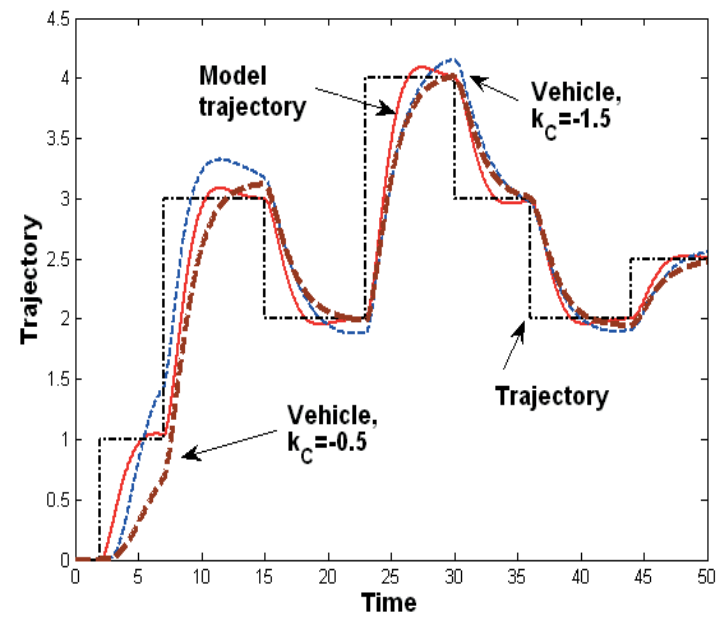

Figure 6. Prescribed trajectory (dash-dot, black), MODEL trajectory (solid, red), VEHICLE trajectory for gain $k_{C}=-1.5$ (dash, blue) and VEHICLE trajectory for gain $k_{c}=0.5$ (dash, brown)

In Fig. 6 is the presented prescribed trajectory, MODEL and VEHICLE trajectory for 2 different gain values of $k_{C}$. It is important to note that for higher values of $k_{C}$ the system can be unstable. 
In Fig. 7 is shown time evolution of parameter $x_{4}$ also for 2 values of $k_{C}$. It can be seen that a big overshoot exists for higher gain $k_{C}$.

So fare the continuous adaptive system was presented. In next part the simulation results for the discrete adaptive system simulations are shown.

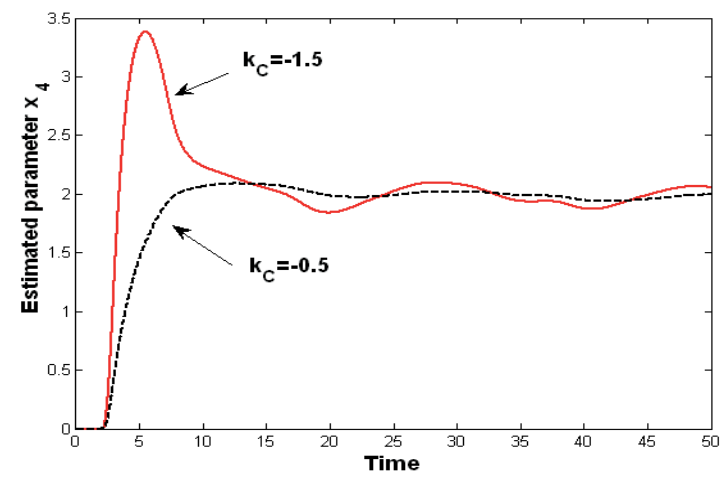

Figure 7. Time evolution of estimated $x_{4}$ for 2 values of $k_{C}$

\section{DigitAl ADAPTIVE CONTROL}

In this part the digital version of adaptive control is described. On the contrary to the continuous adaptive control this approach enables simple changing of parameters or structure of the system. Block diagram of MRAC is shown in Fig. 8. The system is similar to the continuous system (see Fig. 4), the but two analog/digital (A/D) converters and one digital/analog (D/A) converter is used. For the VEHICLE, the continuous transfer function according to eq. (3) is used but the continuous MODEL was converted into discrete version by means of the bilinear transformation

$$
F_{m}(z)=\frac{0.128+0.256 z^{-1}+0.128 z^{-2}}{1-0.767 z^{-1}+0.279 z^{-2}}
$$

Step response of $F_{m}(z)$ is displayed in Fig. 9.

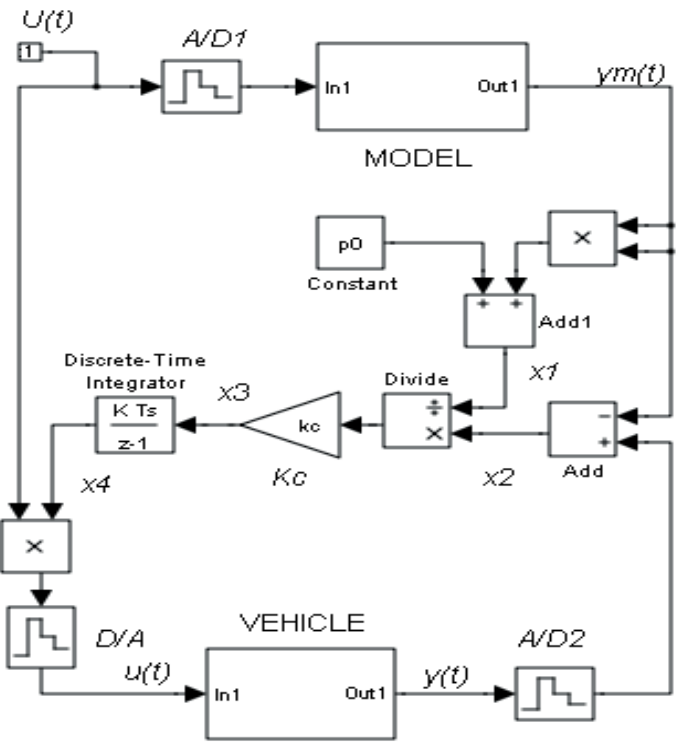

Figure 8. Block diagram of digital adaptive system

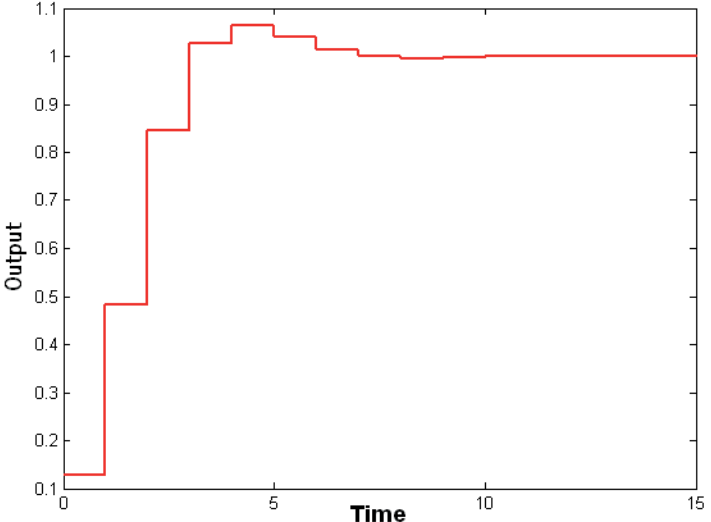

Figure 9. Step response of digital MODEL according $F_{m}(z)$

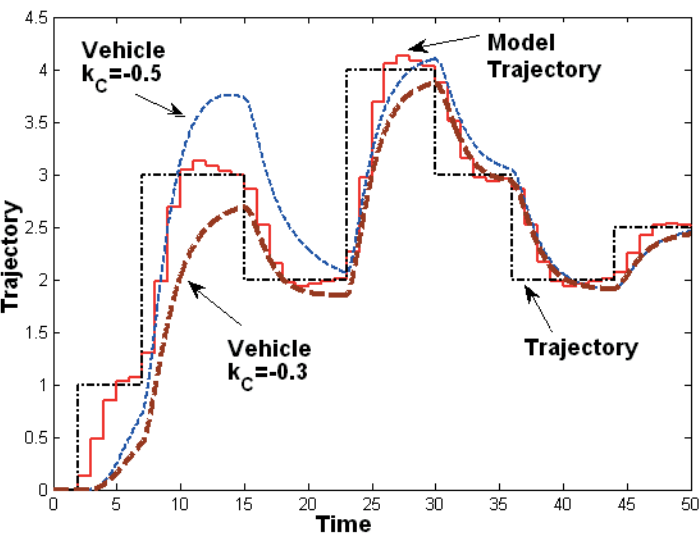

Figure 10. Digital system/ Prescribed trajectory (dash-dot, black) MODEL trajectory (solid, red), VEHICLE trajectory for gain $k_{C}=-$ 1.5 (dash, blue) and VEHICLE trajectory for gain $k_{C}=0.5$ (dash, brown)

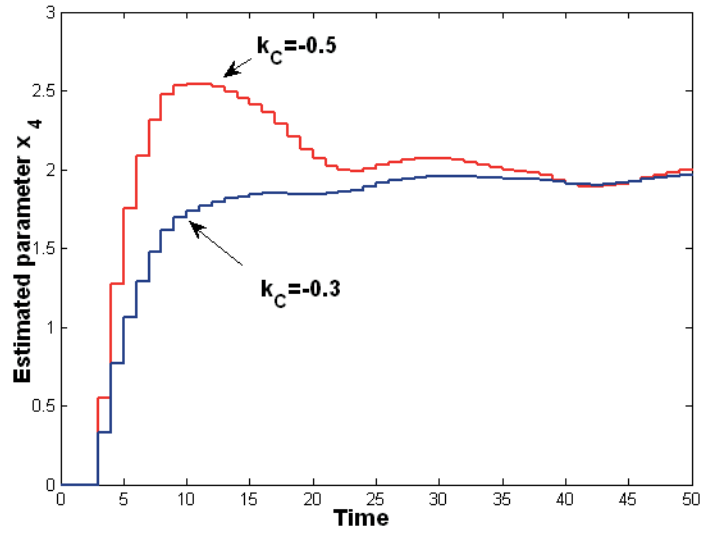

Figure 11. Digital system. Time evolution of estimated $x_{4}$ for 2 values of $k_{C}$

In Fig. 10 is shown the prescribed trajectory, MODEL and VEHICLE trajectory for 2 different values of $k_{C}$. In Fig. 11 is presented the time evolution of parameter $x_{4}$, also for 2 values of $k_{C}$. The overshoot can be seen for higher gain $k_{C}$.

It should be noticed that previous results were derived from the discrete control system, with VEHICLE continuous and all other parts as discrete blocks. 


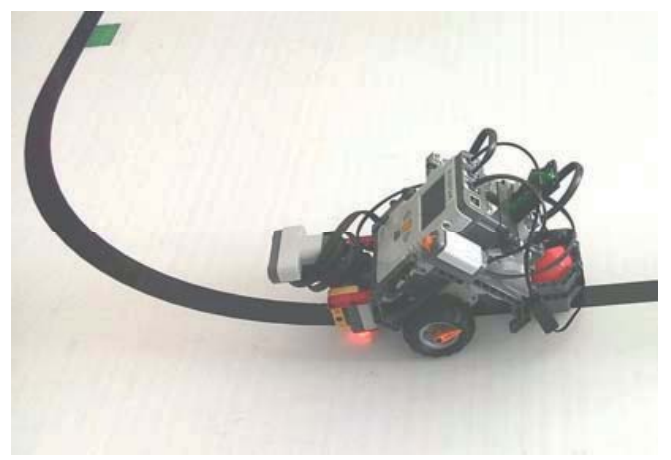

Figure 12. Photo of VEHICLE used for testing of proposed algorithm

In Fig. 12 the photo of VEHICLE used for tests is presented. The VEHICLE has 2 independent 9V DC motors with rotary encoders, the optical sensors and Bluetooth module for communications. Weight is 0.5 $\mathrm{kg}$.

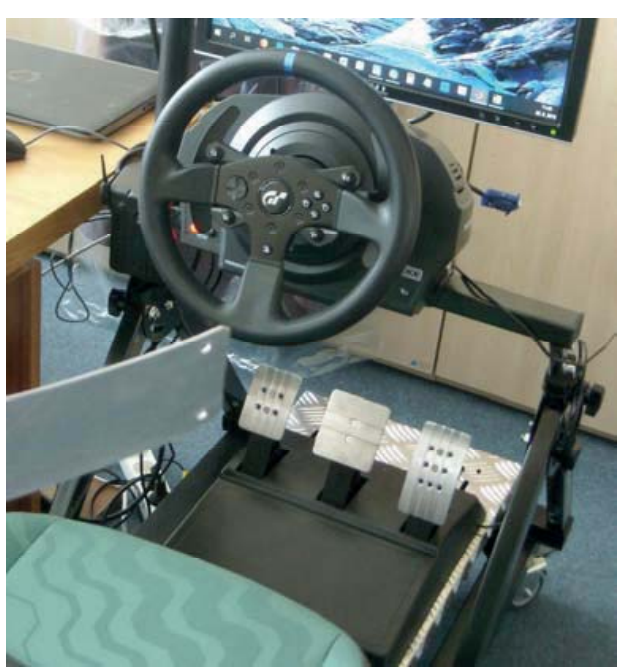

Figure 13. Photo of car driving simulator

For testing purposes the car driving simulator was also developed in department of Applied Electronics. In Fig. 13, the hardware is shown. In Fig. 14, the photo of female subject during test is presented. The infrared sensor $\mathrm{S} 1$ for head position detection is shown. The 2 detectors (on left and right sides) are used.

\section{CONCLUSION}

In this paper the MRAC was described. The theoretical derivations were confirmed by simulations. Two approaches were simulated - continuous and discrete with similar results. Systems, MODELVEHICLE, with different dynamics were deliberately tested. The adaptive algorithms were tested for different gains, because gain value is important for stability and speed of parameter estimation. The car driving simulator was also constructed. The system can be used for further development.

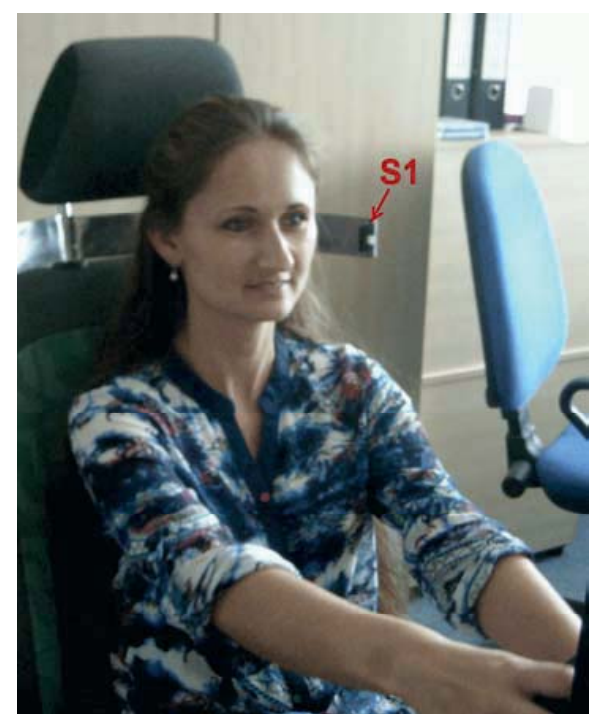

Figure 14. Photo of car driving simulator with infrared distance sensor $\mathrm{S} 1$ for head position detection

\section{REFERENCES}

[1] K. J. Astrom and B. Wittenmark (2001), Adaptive control, 2nd ed., Dover Publications, New York.

[2] Volvo. Autonomous Driving. (http://www.volvocars.com/ int1/about/ourinnovation-brands/intellisafe/autonomousdriving). [Online; 19 January, 2017].

[3] H. Wang, J. Kearney, and K. Atkinson. Arc-length parameterized spline curves for real-time simulation. 2014.

[4] K. Benjelloun, H. Mechlih, E. K. Boukas, "A Modified Model Reference Adaptive Control Algorithm for DC Servomotor", Second IEEE Conference on Control Applications, Vancouver, B. C., Vol. 2, pp. 941 - 946, 1993

[5] M. S. Ehsani, "Adaptive Control of Servo Motor by MRAC Method", IEEE International Conference on Vehicle, Power and Propulsion, Arlington, TX, pp. 78 - 83, 2007.

[6] M. Kirar, P. Swarnkar, S. Jain, R. K. Nema, "Comparative Study of Conventional and Adaptive Schemes for DC Servomotors", International Conference on Energy Engineering ICEE, Puducherry, India, 2009.

[7] P. Swarnkar, S. Jain, R. K. Nema, "Application of Model Reference Adaptive Control Scheme To Second Order System Using MIT Rule", International Conference on Electrical Power and Energy Systems (ICEPES-2010), MANIT, Bhopal, India, 2010.

[8] P. Swarnkar, S. Jain, R. K. Nema, "Effect of Adaptation Gain on System Performance for Model Reference Adaptive Control Scheme Using MIT 